\title{
Research on medical image denoising based on improved median
}

\section{filtering algorithm}

\author{
Wang Jinguo ${ }^{1, a}$, Wang $\mathrm{Na}^{2, b^{*}}$ (corresponding author), Wang rui ${ }^{3, \mathrm{c}}$ \\ ${ }^{1}$ Department of Urology, the First Hospital of Jilin University,China \\ ${ }^{2}$ Department of Anesthesiology, the First Hospital of Jilin University, China \\ ${ }^{3}$ Department of Anesthesiology, the First Hospital of Jilin University, China \\ awangjinguolily@163.com, ${ }^{\mathrm{b}}$ lilyly12345@163.com, ${ }^{\mathrm{C} X}$ Xiaoben6666@126.com
}

Keywords: Medical image processing. Image denoising. Median filtering.

Abstract. Image denoising method can be divided into the spatial and frequency domain processing methods from different processing domains. The former is to deal with it in the two-dimensional space of the image itself. According to the different nature, it can be divided into linear processing method and nonlinear processing method. The latter is used to approximate the original signal function with a set of orthogonal function.

The linear filtering algorithm in spatial domain is more mature, the digital analysis is simple, and the random noise effect is obvious, but it has obvious defects, such as the prior statistical knowledge of random noise, and the poor performance of the image edge details.

\section{Introduction}

The linear filtering algorithm in spatial domain is more mature, the digital analysis is simple, and the random noise effect is obvious, but it has obvious defects, such as the prior statistical knowledge of random noise, and the poor performance of the image edge details[1]. In 1971, Tukey proposed the idea of median filtering, and first applied to the analysis of time series, then this method is introduced to image processing filter image noise, received a good effect. Followed by a variety of median filter improved. One kind of improved algorithm which is called adaptive weighted median filter has attracted people's attention. The most prominent feature of this method is that it has adaptive performance and has a significant improvement on the edge protection ability of the image.

The study of machine vision has made people start to pay attention to the theory of partial differential equations. In this field, the essence of the founding work should be attributed to the work of Koenderink and Witkin. They introduced the theory of scale space in the image, which makes it become the basis of the application of the partial differential equation in the digital image processing. They proposed a partial differential equation with nonlinear filtering ability, and obtained good results in image denoising and edge protection. Later on Weickert based on their theory, this equation developed into an an isotropic diffusion equation, which further improved the denoising ability, and has the effect of the image edge protection which is not possessed by the median filter, mathematical morphology filter and many traditional algorithms. So these methods are widely used in the filtering of medical and remote sensing images in the near future, and satisfactory results are obtained.

With the development and perfection of various theories, digital filtering technology has been 
developed rapidly, and has been widely used in many fields such as medicine, remote sensing, and so on. Now many universities and scientific research institutions have a special medical imaging or Machine Vision Laboratory for this aspect of the technology for a more in-depth study, I believe that with the continuous deepening of research, the update better method will continue to be presented and applied.

\section{Meaning and classification of image noise}

For image noise, it can be defined from two perspectives: one is from the perspective of people's perception, the image noise is the factor that prevents people from recognizing and understanding the image information. The other is from the mathematical point of view, the image information is considered as a spatial function $f$, image noise is the factor that causes the degradation of the information which is expressed by the function $\bar{f}$. Image noise by different methods can be divided into different categories, from the mathematical point of view in order to make the image degradation can be divided into additive noise and multiplicative noise.

If the physical factors of the noise generated by the division, can be divided into the following categories:

1. Electronic noise: noise generated by the electronic movement of the electronic movement in the restive device of the circuit.

2. Photoelectron noise: the image of the photoelectric converter, especially in the condition of weak light, the noise is particularly strong.

3. Photographic particle noise: generally exist in film images. It is because in the film exposure and development, the emulsion of silver halite grains into metallic silver particles shape not and random distribution caused.

4. Speckle noise: in some coherent imaging systems, the noise generated in the image is due to the coherent effects of sound waves and light waves. It also has a relationship with the roughness of the surface of the imaging tissue. Goodman of the irradiate scattered speckle noise properties, Abbott and Thurstone pointed out that the laser spot and ultrasonic speckle interference and the formation of the difference

\section{Median filtering algorithm and improvement}

The basic idea of median filtering is that for an image, a rectangular sliding window is generated at each pixel point in the image, and the average size of the window is taken as an odd number. The median filtering algorithm based on this idea is called the common median filtering algorithm.

Ordinary median filtering algorithm can effectively remove the random noise in the image, but it is not satisfactory for the image edge and details of the protection, the reason is that the uniform method does not have a good edge detail discrimination ability. In view of this, an improved weighted median filtering algorithm is proposed. The idea is as follows: for each pixel $(i, j)$ in the local sliding window, the corresponding weights are calculated:

$$
W(i, j)=[W(N+1, N+1)-a d D / m]
$$

In which, $W(N+1, N+1)$ is the weight of the window center pixel. The a is a constant. The $\mathrm{d}$ is the distant from window center to the point $(\mathrm{i}, \mathrm{j})$. The $\mathrm{D}, \mathrm{M}$ is the window of the variance and average. Then the gray value of the pixel gray value of the formation of a pixel gray level sequence 
$\{g(n)\}$, the weights are adjusted accordingly to form a weight sequence $\{W(n)\}$. Finally select a weight, serial number $\mathrm{i}$, so that the $\mathrm{T}$ is just equal to or greater than the value of the window and the value of the gray value of $g(i)$ as the window of the window to replace the value of the central pixel. This method is called adaptive weighted median filter (AWMF), which has a protective effect on the edge of the image, but it still has some defects. The defects of this algorithm will be analyzed and further improved.

For this adaptive weighted median filter, because the a,d,D,M is more than zero, the weight of the window center point is the largest, and the other pixel points are reduced with the increase of the distance between the center pixel and the $\mathrm{D} / \mathrm{m}$, which is more than the center. The condition of the protection of the edge is $\mathrm{D} / \mathrm{m}$, but it is possible to obtain a large value of $\mathrm{D} / \mathrm{m}$ in the relatively sharp random noise point, which may also have a tendency to keep the noise, which is obviously not what we expect.

In addition, for the $\mathrm{D}, \mathrm{m}$ are small, $\mathrm{m} \mathrm{D/}$ is also very large, making the denoising effect is poor, especially in the black area, M may be 0 , more makes the formula lost meaning. In this method, the parameters of a can only be adjusted by manual adjustment, once the a selection is not properly, it will cause the distortion of the image denoising effect. All of these are the shortcomings of this algorithm, and the improved algorithm will overcome these shortcomings while maintaining the advantages of the above algorithm.

\section{Improved adaptive weighted median filtering algorithm}

Through the above analysis, we realize that in the use of this algorithm, we must have a discriminant of the current sliding window center pixel attribute class standard, we can be classified into three categories: 1 image boundary points or details. 2 image point. 3 image area. While the standard can be used to distinguish the local window of the variance and comply with the following criteria:

Standard 1: if the window of the variance is greater than a certain threshold VT, it is considered that the image of the boundary point or point of view, otherwise it is a regional point.

Standard 2: when the window variance is greater than a certain threshold VT, and then calculate the window outside the window of the central point of the variance, we call this variance for the window to the heart of the variance, denoted as $D^{\prime}$. The absolute value $\left|D-D^{\prime}\right|$ of the difference between the variance of the original window and the window of the window is investigated. If the absolute value is less than half of the current window of $\mathrm{D}$, we think that the central point of the window is the boundary point, otherwise it will be considered as a point mutation.

We have to deal with the different attributes of the above pixel point, and the method can be used to deal with the mutation and the pixels of the region. The method can be used for the average median filter, which only needs a to be 0 , We can give the formula of the A to the center of the weight of the half, and the type (1) re written:

$$
W(i, j)=[W(N+1, N+1)-a d]
$$

So as to obtain the edge protection function, it also makes the processing of different types of pixels to obtain a unified expression form. 


\section{References}

[1] Badulescu P,Zaciu R.Removal of mixed-noise using order statistic filter and wavelet domain Wiener filter. In: Proceedings of the International Semiconductor Conference . 1999.

[2] Jansen M,Bultheel A.Multiple waveletthreshold estimation by generalized cross validation for images with correlated noise. IEEE Transactions on Image Processing . 1999.

[3] A Sawant,H Zeman,D Muratore,etc.An adaptive median filter algorithm to remove impulse noise in X-ray and CT images and speckle in ultrasound images. Proceedings of SPIE the International Society for Optical Engineering . 1999.

[4] Moncef Gabbouj,Edward J. Coyle,Neal C. Gallagher. An overview of median and stack filtering[J]. Circuits Systems and Signal Processing . 1992 (1).

[5] Jin F,Fiegush P,Winger L et al.Adaptive Wiener filtering of noisy images and image sequences. Proceedings of the IEEE International Conference on Image Processing, ICIP 2003.

[6] Jang I H,Kim N C.Locally adaptive Wiener filtering in wavelet domain for image restoration. Proceedings of 1997 IEEE TENCON - Speech and Image Technologies for Computing and Telecommunications . 1997.

[7] Rangsanseri Y,Prasongsook W.Speckle reduction using Wiener filtering in wavelet domain. Proceedings of the 9th International Conference on Neural Information Processing (ICONIP) . 2002. 